\title{
A Three-Dimensional Forward Dynamic Model of the Golf Swing Optimized for Ball Carry Distance
}

\author{
Daniel Balzerson • Joydeep Banerjee • John McPhee
}

Received: date / Accepted: date

\begin{abstract}
A 3D predictive golfer model can be a valuable tool for investigating the golf swing and designing new clubs. A forward dynamic model, which includes a four degree of freedom golfer model, a flexible shaft based on Rayleigh beam theory, an impulse-momentum impact model and a spin rate dependent aerodynamic ball model, is presented. The input torques for the golfer model are provided by parameterized joint torque generators that have been designed to mimic muscle torque production. These joint torques are optimized to create swings and launch conditions that maximize carry distance. The flexible shaft model allows for continuous bending in the transverse directions, axial twisting of the club and variable shaft stiffness as a function of the length. The completed four-part model with the default parameters is used to estimate the ball carry of a golf swing using a particular club. This model will be useful for experimenting with club design parameters to predict their effect on the ball trajectory and carry distance.
\end{abstract}

Keywords Golf Drive · Forward Dynamics · Simulation · Ball Carry · Biomechanics · Modelling

Daniel Balzerson

University of Waterloo, 200 University Ave. W, Waterloo, ON, Canada, N2L 3G1

Tel.: +1-519-888-4567 x33825

Fax: +1-519-4764791

E-mail: dan.johnson@uwaterloo.ca

Joydeep Banerjee

University of Waterloo

John McPhee

University of Waterloo

\section{Introduction \& Background}

\subsection{Motivation}

Computer simulations are used extensively in the design of multibody dynamic systems. In the past they have mostly been used for designing the mechanical and electrical components of systems, but improved techniques have allowed researchers to begin simulating humans interacting with larger systems in biomechatronic models [23]. Simulations of the golf swing have been used since the 1970s [12] to attempt to discover how to play more effectively. By modelling the golfer and club together, we can gain insights into how golfers should swing and the best ways to design their equipment.

Every year there are new claims made by manufacturers about the performance of new clubs. They claim that ball carry distance can be improved by making the club lighter, increasing the moment of inertia of the clubhead, moving the centre of mass of the clubhead, or any number of other factors that may or may not affect the swing. These claims are difficult to evaluate as human tests are not repeatable and robot tests are not completely bio-fidelic. By constructing a validated computer simulation of the golfer and club, it is possible to test the effect of golf club design parameters on the distance the ball can be struck, reliably and repeatably. Some of the questions that the model presented here could answer include: "Do lighter clubs results in longer distances?", "Where should the centre of mass of the clubhead be located?", "How does clubheadm moment of inertia affect driving distance?" and "How long should the shaft of the club be?"

The goal of this project was to develop a golf swing model including the golfer and club that can be evaluated based on its performance in striking the ball. The 
model includes variable parameters for the golfer to allow for optimization of the swing and variable parameters for the golf club to test and evaluate different club designs.

\subsection{Previous Golfer Models}

Since Cochran and Stobbs initial scientific study of the golf swing in 1968 [3], many further attempts to model the golfer and club have been made. Many inverse dynamic (diagnostic) studies have been performed where experimental measurements of golfers are used to drive the model and investigate the swing [26][10][2][37][31]. Fewer forward dynamic, or predictive, models of the swing have been developed.

In 1975, Lampsa used optimal control theory to optimize a torque controlled two-link double-pendulum model of the swing for maximum clubhead speed [12]. Unfortunately, the joint torques produced by that model were dissimilar from those found using inverse dynamics [26]. Pickering and Vickers used the double-pendulum model to examine the release torque at the wrist and found that a natural release of the wrist required the least energy input [29]. More recently, Sharp produced a three-link planar model of the swing that could be optimized by changing parameterized input torques to produce the fastest clubhead speeds [34].

MacKenzie's model from 2009 also used the concept of parameterized joint torques to improve the feasibility of optimizing the golf swing [15]. His model introduced a fourth degree of freedom to the model golfer allowing for 3-dimensional motion through the supination and pronation of the forearm. This model also included a simple flexible shaft model by dividing the shaft into four rigid sections with a spring and damper at each joint.

There remain significant opportunities for creating an improved golfer model that allows for new questions to be asked and answered. The model presented in this paper incorporates many of the features from previous models along with the following novel elements: a golfer model including active and passive joint torques designed to mimic human muscle; a flexible shaft model that allows for continually varying stiffness parameters along the length of the shaft; and evaluation of each swing using an impact and aerodynamic ball trajectory model.

\section{Golf System Model}

To simulate the golfer and club and evaluate swings based on ball carry distance, a four-part model of the

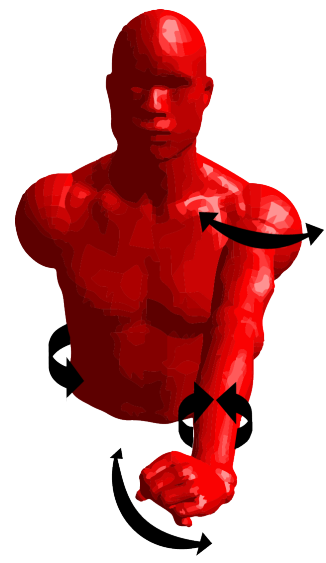

Fig. 1 Golfer model with four degrees of freedom indicated

golf swing was constructed. This section will describe each part, its implementation, and its validation.

\subsection{Golfer}

The golfer portion of the mathematical model consists of three rigid bodies representing the torso, left arm, and left hand of the golfer. There are four degrees of freedom (DoF) for the golfer. The first DoF is the rotation of the torso. This represents the rotation of the shoulders during the golf swing and is activated by the power of the muscles of the legs and core. The second DoF allows transverse flexion and transverse adduction of the arm across the front of the body. The third DoF allows supination and pronation of the forearm and the final DoF allows for ulnar and radial deviation of the wrist. These four DoF are illustrated in Figure 1. This golfer model, based on the work of MacKenzie [15] was considered to be sufficient to apply the correct kinetics to the club shaft throughout the swing.

The mass and inertia properties for the torso and arm of the golfer were taken from the work of MacKenzie [14] and are shown in Table 1. The mass and moment of inertia of the hand also takes into account the mass of the grip of the shaft and is also shown in Table 1 . The segment geometries are shown in Table 2. Finally, the golfer's torso was inclined 30 degrees from the vertical and the swing plane of the arms was inclined 50 degrees from the horizontal. Separate planes of rotation for the shoulder and arm rotation are better able to mimic the swing of a human golfer than single-plane models [8].

\subsubsection{Active Torque Inputs}

The input torques for the golfer model were defined by the same functions used by MacKenzie [16], taking into account both the activation and force-velocity curves 
Table 1 Segment mass properties of the golfer.

\begin{tabular}{ccccc} 
Segment & Mass $(\mathrm{kg})$ & $I_{x x}\left(\mathrm{~kg} \mathrm{~cm}^{2}\right)$ & $I_{y y}\left(\mathrm{~kg} \mathrm{~cm}^{2}\right)$ & $I_{z z}\left(\mathrm{~kg} \mathrm{~cm}^{2}\right)$ \\
\hline Torso & 34.61 & $\ldots$ & 3655 & $\ldots$ \\
Arm & 3.431 & 1076 & 1096 & 58.06 \\
Hand \& Grip & 0.6 & 10.24 & 10.24 & 6.04 \\
\hline
\end{tabular}

Table 2 Segment geometry properties of the golfer.

\begin{tabular}{ccccc} 
Segment & Length $(\mathrm{cm})$ & $C M L o c_{x}(\mathrm{~cm})$ & $C M L o c_{y}(\mathrm{~cm})$ & $C M L o c_{z}(\mathrm{~cm})$ \\
\hline Torso & 20 & 0 & 0 & 0 \\
Arm & 60 & 0 & 0 & 26.1 \\
Hand \& Grip & 20 & 0 & 0 & 9.0 \\
\hline
\end{tabular}

of human muscles. Each torque generator is controlled by 5 parameters as shown in Table 3 . The arm generator provides supination/pronation torques about the long axis of the arm while the shoulder generator provides torque to rotate the arm in the swing plane. The generated torque is calculated as follows.

First, the pre-scaled torque, $T_{\text {pre }}(t)$, is calculated using

$T_{\text {pre }}(t)=T_{m}\left(1-e^{\frac{t_{o n}}{\tau_{a}}}\right)-T_{m}\left(1-e^{\frac{t_{o f f}}{\tau_{d}}}\right)$

where $T_{m}$ is the maximum possible applied torque, $\tau_{a}$ is the time constant of activation, and $\tau_{d}$ is the time constant of deactivation. The functions $t_{\text {on }}$ and $t_{\text {off }}$ are the amount of time that has passed since the torque was activated and deactivated, respectively, and are calculated as piecewise ramp functions:

$t_{\text {on }}(t)= \begin{cases}0 & : t<t_{a} \\ t-t_{\text {activate }} & : t>t_{a}\end{cases}$

$t_{o f f}(t)= \begin{cases}0 & : t<t_{d} \\ t-t_{\text {deactivate }}: t>t_{d}\end{cases}$

where $t_{a}$ is the time at which the joint torque is activated and $t_{d}$ is the time at which it is deactivated.

Then, the value of $T_{\text {pre }}(t)$ is scaled based on the fact that muscles cannot exert as much torque on limbs that are already moving quickly. As the angular speed $(\omega)$ of the segment increases, the torque provided is decreased based on the following scaling:

$T(t, \omega)=T_{\text {pre }}(t) \frac{\omega_{\max }-\omega}{\omega_{\max }+\Gamma \omega}$

This approach was selected because it accounts for both the activation dynamics and the force-velocity relationship for the muscles [27] while keeping the number of control parameters small. Since the maximum torque, activation constants, and shape parameters remain constant across swings, only the activation timings need to be determined for each torque generator in the model. This results in 8 muscle parameters that must be chosen during the optimization process, two for each DoF.

\subsubsection{Passive Joint Torques}

As an extension to the model proposed by Mackenzie, passive joint torques are included for the torso, shoulder, and wrist that represent the energy stored during the backswing. These moments model the passive forces applied by the elastic tissue surrounding the joint at the limits of the range of motion. To model this passive torque, Yamaguchi [38] proposed the use of (5).

$T_{\text {passive }}(\theta, \dot{\theta})=k_{1} e^{-k_{2}\left(\theta-\theta_{-}\right)}-k_{3} e^{-k_{4}\left(\theta_{+}-\theta\right)}-c_{1} \dot{\theta}$

This function is able to approximate the restoring moment at both extremes of the joint range of motion and offer a smooth transition in joint torque from the normal range of motion, where very little torque is applied, to the large moments applied at the edges. The constants $k_{1}$ and $k_{3}$ govern the magnitude of the force at the breakpoints $\left(\theta_{-}\right.$and $\left.\theta_{+}\right)$while $k_{2}$ and $k_{4}$ govern the sharpness of the break. For this form, $\theta_{-}$and $\theta_{+}$ should be set well within the range of motion of the joint.

For each joint with a passive component, the values $k_{1}, k_{2}, k_{3}, k_{4}, \theta_{-}$and $\theta_{+}$must be found. A careful search of the literature found explicit values of these parameters for ankle, knee, and hip moments [38] [18] [1] but no values for the upper body were found. Instead, the parameters were determined from experimental data [5] [7] [21]. The extracted parameters are given in Table 4. A sample of the fitted curve plotted against the experimental for the shoulder data is shown in Figure 2.1.2

\subsubsection{Control of the Golfer}

By replacing the golfer's muscle dynamics with parameterized joint torque functions in the form proposed by MacKenzie [17], control of the swing can be achieved by selecting appropriate values for $t_{\text {activate }}$ and $t_{\text {deactivate }}$ in (2) and (3). It is assumed that the swing is quick enough that the golfer cannot turn their muscles on and off multiple times during the swing and that the golfer attempts to swing with maximum power. By modifying 
Table 3 Parameters for the four active joint torque generators.

\begin{tabular}{ccccccc} 
Generator & $T_{m}(\mathrm{Nm})$ & $\tau_{a}(\mathrm{~s})$ & $\tau_{d}(\mathrm{~s})$ & $\omega_{\max }\left(\mathrm{rad} \mathrm{s}^{-1}\right)$ & $\Gamma$ \\
\hline Torso & 200 & 0.02 & 0.04 & 30 & 4.0 \\
Shoulder & 160 & 0.02 & 0.04 & 30 & 4.0 \\
Forearm & 90 & 0.02 & 0.04 & 60 & 4.0 \\
Wrist & 90 & 0.02 & 0.04 & 60 & 4.0 \\
\hline
\end{tabular}

Table 4 Parameters for passive joint torques at the torso, shoulder, and wrist joints.

\begin{tabular}{cccccccc} 
Joint & $\theta_{-}(\mathrm{rad})$ & $\theta_{+}(\mathrm{rad})$ & $k_{1}$ & $k_{2}$ & $k_{3}$ & $k_{4}$ & $c_{1}$ \\
\hline Torso & 0.0618 & -0.693 & 3.898 & 2.082 & 3.814 & 2.098 & 0.1 \\
Shoulder & -1.289 & 1.210 & 2.111 & 3.354 & 2.704 & 2.241 & 0.1 \\
Wrist & -1.171 & 1.185 & 4.301 & 2.732 & 3.895 & 2.891 & 0.1 \\
Forearm & -1.237 & 1.340 & 3.206 & 2.624 & 2.216 & 1.752 & 0.1 \\
\hline
\end{tabular}

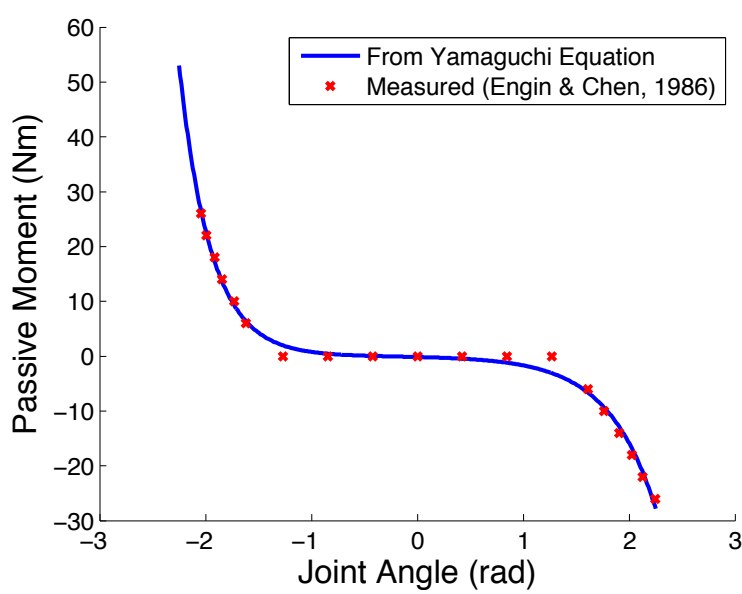

Fig. 2 Passive torque for the shoulder joint. The measured experimental torques are marked by crosses and the fitted curve is shown as a solid line.

the relative timings of the joint torque activations, different swings can be achieved. The process for selecting the optimal swing parameters for a particular club will be discussed in detail in Section 2.5.

\subsubsection{Validation}

The golfer model was validated using data from MacKenzie by using the model to generate a swing similar to that of a human golfer [15]. The model was found to produce angular displacement curves for the torso, shoulder, arm, and club that well matched the swing of the real golfer given the same initial starting configuration. This matching was performed by using the 8 control parameters for the joint torques along with a scale factor for the maximum joint torque values and angular velocities for each joint. Mackenzie's experiments showed that the four degree of freedom golfer model was able to adapt to match the swings of a human golfer through varying the control parameters. We compared the mag-

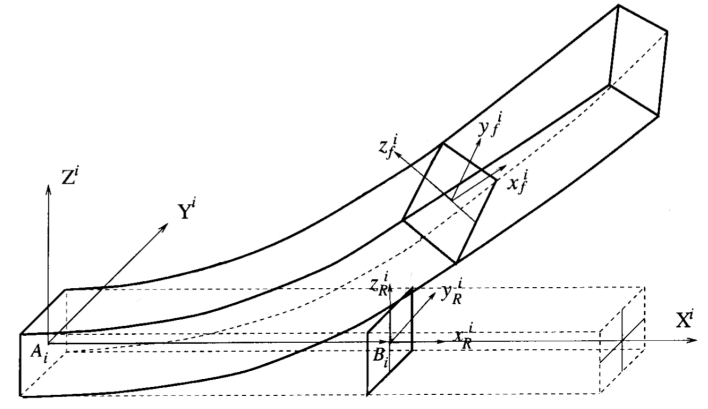

Fig. 3 Flexible beam model as proposed by Shi et al. [35]

nitude of the simulated passive forces to the values found in [5] [7] [21] and found good agreement.

\section{$2.2 \mathrm{Club}$}

\subsubsection{Flexible Shaft}

The club model consists of two parts, the flexible shaft and the clubhead. The flexible shaft used in the model is based on the work of Sandhu et al. and a detailed description can be found in [32]. The model uses a flexible Rayleigh beam [35] to describe the flexing and twisting of the club as it is swung. The approach makes use of a complete second-order elastic rotation matrix for a Rayleigh beam and has been implemented in the simulation package MapleSim. Shear due to bending is neglected, but the model can account for large deflections in the transverse directions and torsion about the shaft that occur during the golf swing. The model can also account for changing stiffness, size, and density of material along the length of the shaft by defining each as a function of the distance from the bottom of the grip, $x$. Figure 3 shows the types of deformations that can be modeled using this type of beam.

The parameters for the flexible shaft, $E$ (stiffness), $I$ (area moment of inertia), $G$ (torsional stiffness), $J$ (po- 


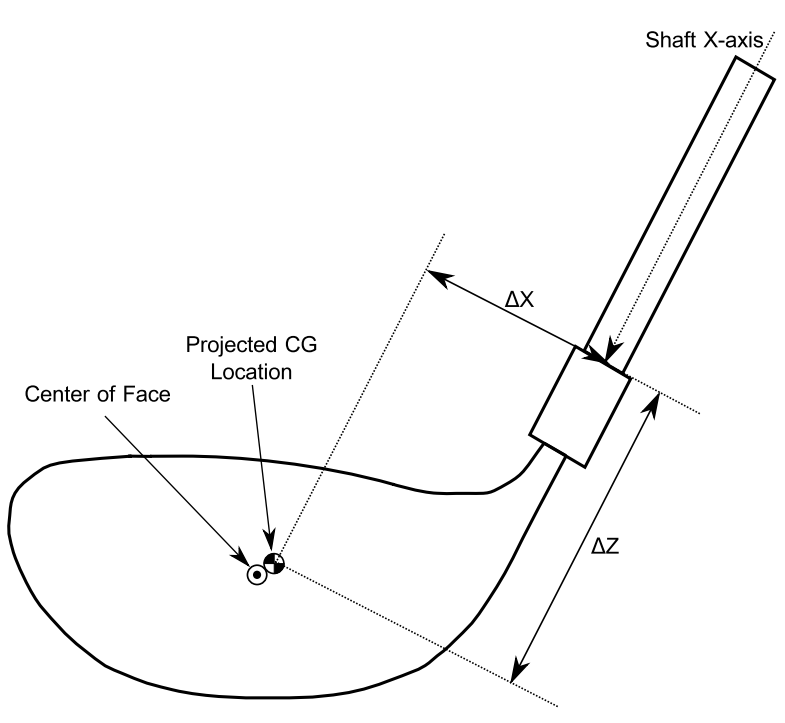

Fig. 4 Front view of clubhead model.

lar moment of inertia), and $A$ (cross-sectional area) are approximated using sixth-order polynomial functions of $x$, the distance from the grip. For this work, stiffness for the flexible shaft was provided by a clubhead manufacturer and polynomials fitting each of the manufacturers curves were estimated. The shaft has higher stiffness near the grip and lower stiffness near the clubhead.

\subsubsection{Clubhead}

The clubhead is modeled as a rigid body fixed to the end of the flexible shaft. The important parameters for the clubhead are the mass, the location of the centre of mass, and the moment of inertia of the clubhead about the vertical axis. These properties were measured for a set of clubheads as part of a different project and one clubhead was selected for initial use in this work. The properties of the selected clubhead are shown in Table 5. To interpret the location of the centre of mass, Figures 4 and 5 show the corresponding frames of reference. The moment of inertia of the club was measured in a reference frame with the horizontal $\mathrm{x}$-axis out of the face of the club, the vertical y-axis upwards at address position, and the z-axis away from the golfer (see $x_{c} y_{c} z_{c}$ in Figures 8 and 9 ).

Table 5 Clubhead geometry and mass properties

\begin{tabular}{cccc}
$\Delta X(\mathrm{~mm})$ & $\Delta Y(\mathrm{~mm})$ & $\Delta Z(\mathrm{~mm})$ & Mass $(\mathrm{g})$ \\
\hline 40.42 & 13.2 & 56.0 & 200 \\
\hline
\end{tabular}

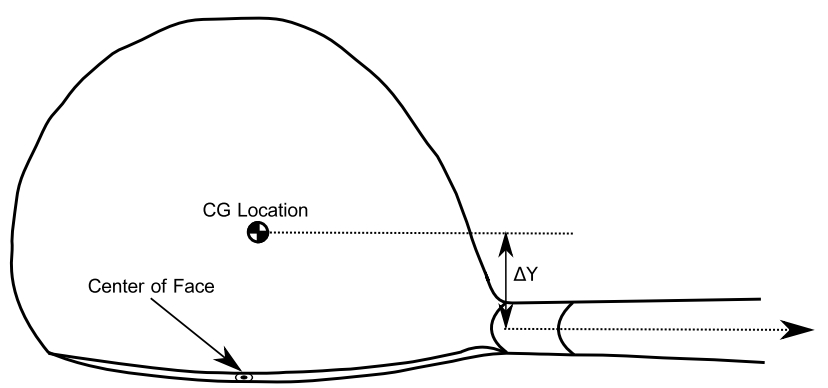

Fig. 5 Top view of clubhead model.

\subsubsection{Club Aerodynamics}

The aerodynamics of the clubhead have a small, but not insignificant effect on the swing. Recently, several golf club companies have claimed that they are able to reduce the drag on their clubheads through the addition of small turbulators and other features that change the way the airflow affects the club [6]. To account for aerodynamic effects, drag on the clubhead is included in the model using the standard drag equation:

$\mathbf{F}_{\mathbf{d}}=-\left(\frac{1}{2} \rho A C_{d}\left|\mathbf{V}_{\mathbf{c}}\right|^{2}\right) \hat{\mathbf{V}}_{\mathbf{c}}$

where $\rho$ is the density of the air, $A$ is the cross-sectional area of the clubhead, and $C_{d}$ is coefficient of drag of the clubhead.

Measurements of $C_{d}$ were provided from experiments performed in a wind tunnel. A club was placed in the tunnel and rotated from a heel-first presentation to a face-first presentation at a variety of wind speeds. The $C_{d}$ value for the clubhead was found to vary with both the presentation angle of the clubhead and the wind speed. The $C_{d}$ values for each yaw angle at high clubhead speeds (greater than $33.5 \mathrm{~m} \mathrm{~s}^{-1}$ ) are shown in Table 6 . At lower speeds, $C_{d}$ was found to be $50 \%$ higher at $22.5 \mathrm{~m} \mathrm{~s}^{-1}$. The transition zone between high speeds and low speeds is approximated linearly. Figure 6 shows a linear interpolation of the values of $C_{d}$ for a range of values of the yaw angle and clubhead speed.

Table 6 Measured values of $C_{d}$ for a variety of yaw angles at high clubhead speeds. A yaw angle of 0 degrees corresponds to a heel-first clubhead presentation (e.g., top of the backswing) while a yaw angle of 90 degrees corresponds to a face-first presentation (e.g., at impact).

\begin{tabular}{cc} 
Yaw Angle (deg) & $C_{d}$ \\
\hline 0 & 1.10 \\
10 & 0.76 \\
30 & 0.74 \\
50 & 0.82 \\
70 & 0.57 \\
80 & 0.51 \\
90 & 0.55 \\
\hline
\end{tabular}




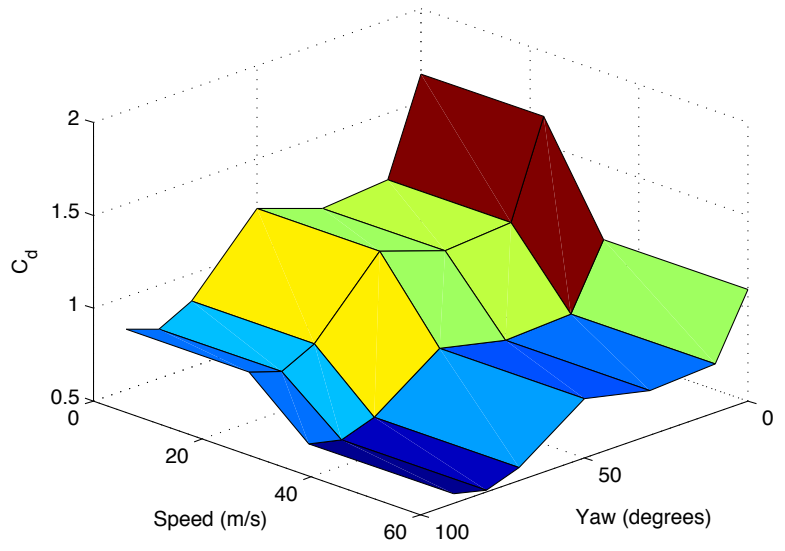

Fig. $6 C_{d}$ values for the clubhead as modeled for different combinations of clubhead speed and yaw angle.

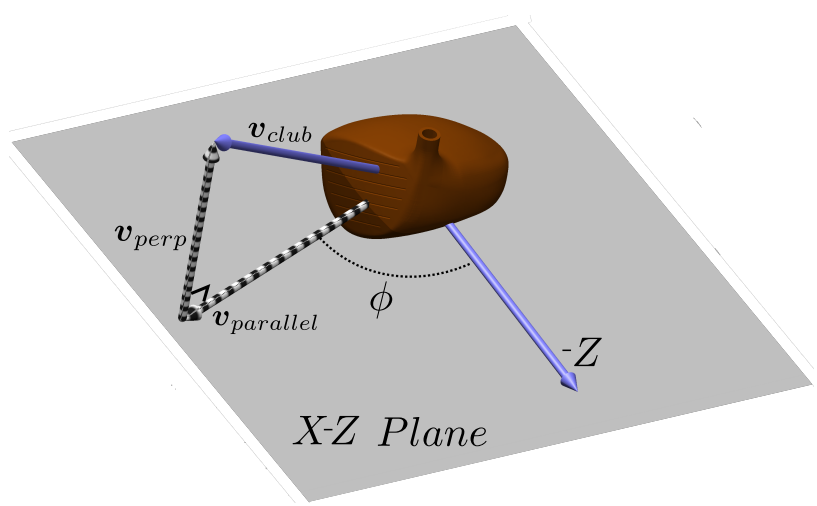

Fig. 7 An illustration of the relevant frames and velocities for calculating the aerodynamic loads on the clubhead.

Since the $C_{d}$ value is dependent on both the yaw angle and the velocity of the clubhead relative to the air, we need to define these two variables within the context of the model. This requires the definition of a new coordinate system which is body-fixed in the clubhead with the $\mathrm{X}$-axis pointing out of the face, the $\mathrm{Y}$-axis upward along the shaft of the club, and the Z-axis tangent to the club face. We use this coordinate system to define the $X-Z$ plane in which the yaw angle of the club is calculated. To calculate the yaw angle, the velocity of the club is split into two components, $\mathbf{v}_{p}$ in the $X-Z$ plane, and $\mathbf{v}_{y}$ normal to the plane. The yaw angle $(\phi)$ is the angle between $\mathbf{v}_{p}$ and the $-Z$ axis. The speed of the airflow used in the calculation of $\mathbf{F}_{d}$ is then $\mathbf{v}_{p}$. Figure 7 illustrates the both the yaw angle and $\mathbf{v}_{p}$. The value of $C_{d}$ at each moment is determined using Figure 7 and the current values of $\phi$ and $\left|\mathbf{v}_{p}\right|$. Using this information, we can rewrite our aerodynamic equation as

$\mathbf{F}_{d}=-\left(\frac{1}{2} \rho A C_{d}\left(\phi,\left|\mathbf{v}_{p}\right|\right)\left|\mathbf{v}_{p}\right|^{2}\right) \hat{\mathbf{v}}_{p}$

Finally, the effective cross-sectional area of the club was provided as a constant $A=0.004805 \mathrm{~m}^{2}$ and the density of the air used in the simulations was $\rho=1.1839$ $\mathrm{kg} \mathrm{m}^{-3}$.

\subsubsection{Validation}

The flexible shaft model was validated using data from Sandhu et al [32]. Sandhu performed a motion capture experiment with four golfers in order to capture both the grip kinematics and the motion of the clubhead. This experiment captured both grip and clubhead kinematics of four subjects in order to validate the model of the flexible club. The grip kinematics were then given to the flexible model of the club and the dynamic loft, droop, and clubhead speed compared between the analytical model, a finite element model, and the experimental data. The analytical model was able to achieve good agreement with the finite element model throughout the swing and good agreement with the experimental results during the impact phase of the swing. The modelled dynamic loft, droop, and clubhead speed at impact were found to be within $10 \%$ of the experimental results [32]. The clubhead aerodynamics were compared to experimental data from [6] and found to be in good agreement with the modelled drag force peaking at $6 \mathrm{~N}$ compared to wind tunnel measurements of $6.5 \mathrm{~N}$ to $9 \mathrm{~N}$.

\subsection{Impact}

The role of the impact model in this work was to calculate the ball launch conditions based on the clubhead velocity, orientation, and angular velocity at impact. The impact model should be realistic, resulting in slice and hook shots for hits with non-ideal clubhead conditions. It was important that the impact model simulate quickly as the impact and aerodynamics portion of the model must be used many times within each simulation to determine the optimal timing for striking the ball.

The impact model selected was based on the work of Petersen and McPhee [28] and is a three-dimensional impulse-momentum approach. In calculating the impact, the ball and clubhead each have 6 degrees of freedom and therefore have 6 velocity components following the impact. The three impulses of the impact are also unknown and must be determined, leading to a total of 15 unknowns.

Figure 8 and Figure 9 shows the free body diagram and frames of reference used for developing the impulse 
and momentum equations for the clubhead and the ball. There are four relevant reference frames. The first is the global frame $(X, Y, Z)$ in which the clubhead velocity is determined from the swing model and the ball velocity is calculated. For this frame, $X$ is the downrange direction, $Y$ is upwards, and $Z$ is outwards away from a right handed golfer. The second reference frame $\left(x_{c}, y_{c}, z_{c}\right)$ is the clubhead frame which is coincident with the global frame when the club is at address position and is body fixed in the club at the centre of mass. The clubhead moments of inertia are defined in this frame. The third reference frame is the ellipsoid frame $\left(x_{e}, y_{e}, z_{e}\right)$ which is used to define an analytical shape for the face of the clubhead. This frame has its origin at the center of an ellipsoid defined by the clubhead's bulge and roll and is inclined from the clubhead frame by the loft angle $(\alpha)$ of the club so that the $x_{e}$ axis passes through the centre of face of the club normal to the surface. The final frame of reference is the impact frame $\left(x_{i}, y_{i}, z_{i}\right)$ which is normal to the clubface at the point of impact. The angles $\gamma$ and $\beta$ between the ellipsoid frame and the impact frame are caused for an off-centre impact by the bulge and roll of the club and are calculated using the ellipsoid which approximates the surface of the clubface.

The system equations are resolved in the impact frame. By applying the principles of impulse and momentum to the bodies involved, the 12 equations for the clubhead and ball can be written as

$m_{c} \mathbf{V}_{c}-m_{c} \mathbf{v}_{c}=-\mathbf{P}$

$\mathbf{I}_{c} \cdot \boldsymbol{\Omega}_{c}-\mathbf{I}_{c} \cdot \boldsymbol{\omega}_{c}=\mathbf{r}_{i m p} \times-\mathbf{P}$

$m_{b} \mathbf{V}_{b}-m_{b} \mathbf{v}_{b}=\mathbf{P}$

$\mathbf{I}_{b} \cdot \boldsymbol{\Omega}_{b}-\mathbf{I}_{b} \cdot \boldsymbol{\omega}_{b}=\mathbf{r}_{b} \times \mathbf{P}$.

In these equations, capital letters stand for the velocity and spin of the ball and club after impact and lowercase letters will be used for the velocity and spin before impact. $m_{c}, \mathbf{I}_{c}, m_{b}$, and $\mathbf{I}_{b}$ represent the mass and inertia tensors for the club and ball respectively. $\mathbf{P}$ is the combined vector of the three impulses, $\mathbf{P}_{n}, \mathbf{P}_{z}$, and $\mathbf{P}_{y}$. $\mathbf{r}_{i m p}$ is the vector from the center of mass of the club to the impact point in the impact frame, and $\mathbf{r}_{b}$ is the vector from the center of mass of the ball to the impact point in the impact frame.

Three further equations are required to solve for the 15 unknowns. First, assuming the ball rolls without slipping on the face of the club, we have two kinematic constraints that restrict the point of contact on the ball and club to be moving in the same direction at the same speed after impact.

$V_{c_{y}}-V_{b_{y}}=0$
$V_{c_{z}}-V_{b_{z}}=0$

And finally we have the coefficient of restitution equation which accounts for energy lost during the impact due to the deformation of the ball and the vibration of the clubhead.

$e=\frac{V_{c_{x}}-V_{b_{x}}}{v_{c_{x}}-v_{b_{x}}}$

Solving all 15 equations simultaneously, the ball spin and velocity after impact can be found. The required parameters for the impact model can be found in Table 7 .

Table 7 Required clubhead and ball parameters for the impact model.

\begin{tabular}{lc} 
Parameter & Value \\
\hline$m_{c}(\mathrm{~g})$ & 200 \\
$I_{c_{x x}}\left(\mathrm{~g} \mathrm{~cm}^{2}\right)$ & 3000 \\
$I_{c_{y y}}\left(\mathrm{~g} \mathrm{~cm}^{2}\right)$ & 4200 \\
$I_{c_{z z}}\left(\mathrm{~g} \mathrm{~cm}^{2}\right)$ & 2200 \\
Bulge $(\mathrm{cm})$ & 30 \\
Roll $(\mathrm{cm})$ & 30 \\
$m_{b}(\mathrm{~g})$ & 45.93 \\
$r_{b}(\mathrm{~cm})$ & 2.13 \\
$I_{b}\left(\mathrm{~g} \mathrm{~cm}^{2}\right)$ & 83.35 \\
\hline
\end{tabular}

\subsubsection{Validation}

Validation of the impact model was performed as part of the work of Petersen and McPhee [28]. In this work, the results of impulse-momentum impact model were compared to the results from a finite element model of the ball and club impact. For an impact at the sweet spot of the club, the velocity of the ball after impact was within $6 \%$ of the finite element model. Additionally, the impact model was compared to results from robot testing performed by Golf Labs. Using impact data from center hits on 4 different clubheads, the impact model was found to overestimate the amount of spin on the ball after impact by an average of $9 \%$ or about 250 RPM. This is due to the assumption that the ball rolls without slipping on the face of the club. Ball-slip would reduce the gear effect and reduce the spin of the ball. The ball launch velocity was underpredicted by an average of $3.5 \%$ or about $5 \mathrm{mph}$.

\subsection{Ball Trajectory Model}

After the ball launch velocity and spin has been calculated, the ball flight is computed using a trajectory model to allow for comparisons of impacts. By using a trajectory model, it is possible to evaluate the outcome 


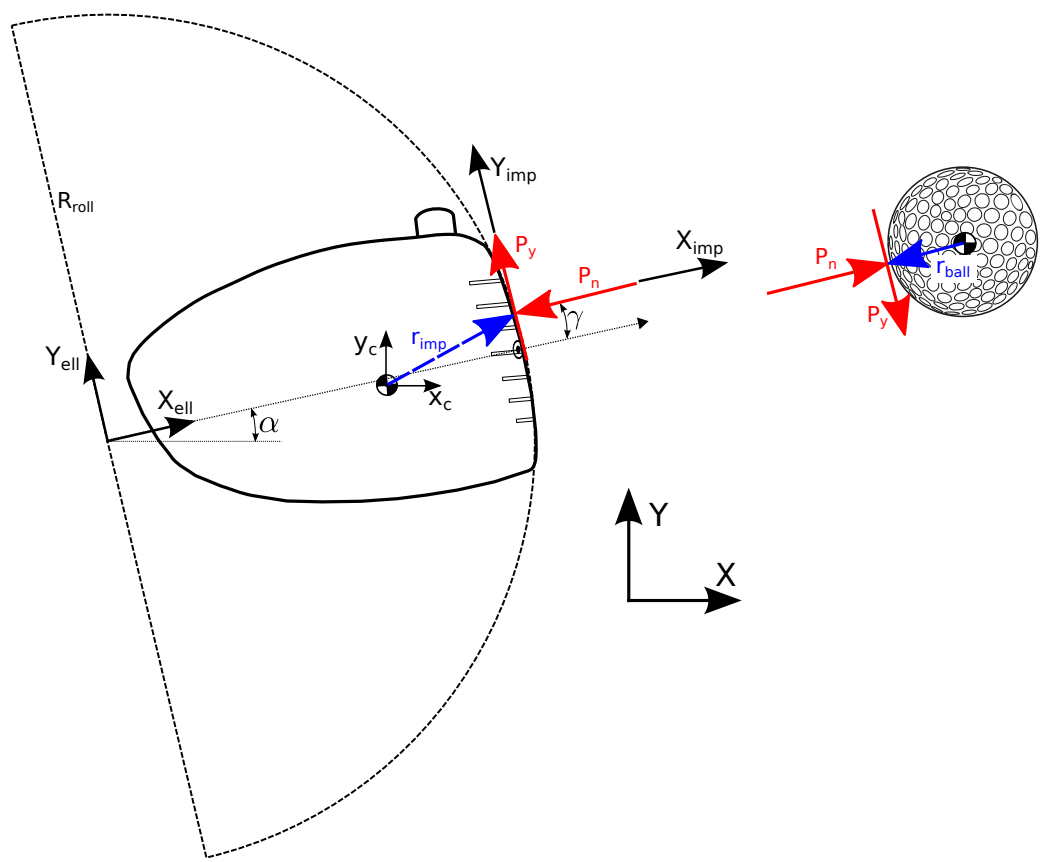

Fig. 8 Side view of the impact model illustrating impulses, frames of reference, and the clubhead ellipsoid.

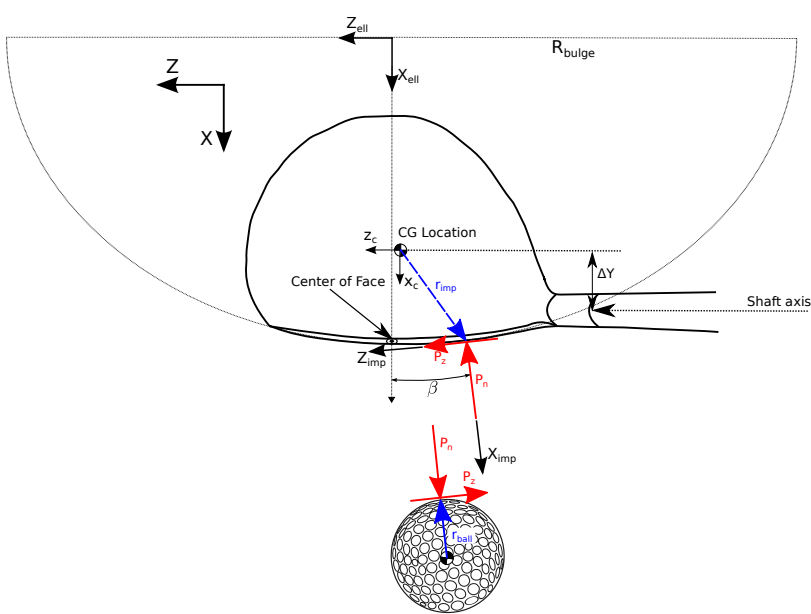

Fig. 9 Top view of impact model illustrating impulses, frames of reference, and the clubhead ellipsoid.

of a golf swing based on an intuitive measure of its success: the distance the ball travels. This model takes into account the lift, drag, and gravitational forces on the ball in flight. It also includes a decay term for the spin of the ball. A free body diagram of the ball in flight is shown in Figure 10. The aerodynamic model used is based on the work of Quintavalla [30] which provides equations and coefficients for calculating the forces on the ball in flight. It also includes the ability to include wind conditions and elevation data for the tee, but these factors were not included in the model.

The model is simple and uses the usual aerodynamic equations for calculating the lift and drag forces on the

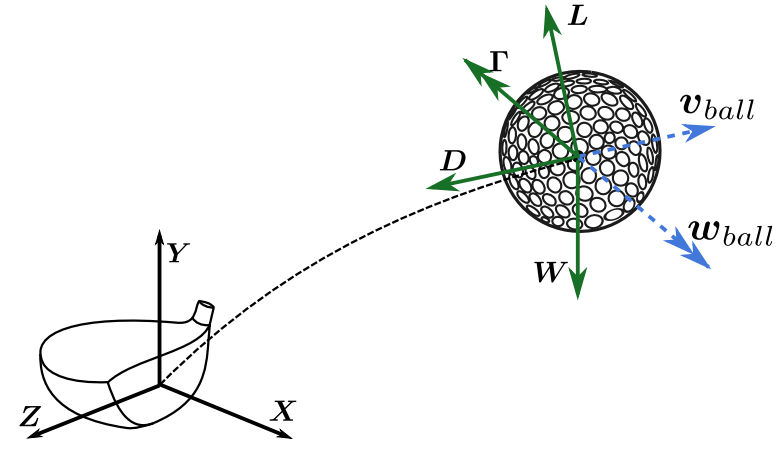

Fig. 10 Free body diagram of the ball in flight.

ball [22]. The coefficients $C_{D}, C_{L}$, and $C_{M}$ are determined experimentally and the values were found to be dependent on the spin rate of the ball $S_{p}$.

$S_{p}=\frac{\omega_{b a l l} \frac{D}{2}}{V_{b}}$

And the coefficients calculated as follows:

$$
\begin{aligned}
C_{D} & =0.171+0.62 S_{p} \\
C_{L} & =0.083+0.885 S_{p} \\
C_{M} & =0.0125 S_{p}
\end{aligned}
$$

where $D$ is the diameter of the ball, and $V_{b}$ is the speed of the ball. These values were given in imperial units so a unit conversion was performed before the aerodynamic calculations were performed. 
Once the values of the aerodynamic coefficients were determined, the equations of motion for the ball were found by projecting the force equations onto the global coordinate system. The resulting equations of motion for the ball are numerically integrated within Matlab using an explicit Runge-Kutta(4,5) formula to determine the ball trajectory.

\subsubsection{Validation}

Validation of the ball trajectory model was performed by comparing the results to robot testing data. In this comparison, ball launch conditions from 10 different swings were entered into the aerodynamic model and compared to their actual trajectories from robot testing. The mean carry distance was found to be $3.23 \mathrm{~m}$ less than the robot testing data with the mean dispersion distance being only $0.36 \mathrm{~m}$ different. The ball model used in this work is from older ball data and could be updated to include modern coefficients if they were available. This would help to resolve the discrepancy between the model and the robot testing results.

\subsection{Optimal Control}

In order for the simulated golfer to adapt to different situations, it is important to control the swing to produce the best ball carry for each set of simulation parameters used. A human golfer would modify their swing for different clubs, and the simulated golfer should similarly adjust. The optimal control of the golf swing is a difficult problem to solve directly as there are many inputs and biological constraints on the inputs; instead of using conventional optimal control techniques (e.g., Pontryagin's Minimum Principle or dynamic programming) the control of the model was achieved by the selection of parameters for the muscle torque generators.

The optimal control of the golfer model was performed through the activation and deactivation timing of the four torque generators in the biomechanical model. From (1) and (3), each torque generator is controlled by the timing parameters $t_{\text {activate }}$ and $t_{\text {deactivate }}$. Through the selection of these parameters, the optimal control of the swing is reduced from a free optimal control problem with arbitrary torques throughout the duration of the swing to a constrained parameter optimization problem.

The objective function is designed to use the most intuitive method for evaluating a swing: by examining the flight path of the ball. The goal is to maximize the distance the ball carries while minimizing the lateral deviation of its flight. The chosen function allows for a small amount of lateral deviation without a significant penalty to simulate the ball landing in the fairway, but larger deviations are heavily penalized to simulate landing in the rough or out of bounds. The equation for the objective function is

$M=X-W e^{Z^{2} / Z_{\max }^{2}}$

where $X$ is the downrange carry, $Z$ is the lateral deviation, $Z_{\max }$ is the maximum acceptable deviation, and $W$ is a weighting term. The value of $Z_{\max }$ was chosen to be $4.57 \mathrm{~m}$ ( 5 yards) and the value of $W$ to be $9.14 \mathrm{~m}$ (10 yards).

\subsubsection{Striking the Ball}

One important question remains in choosing the optimal parameters for the swing: "Where should the ball be placed by the golfer?" or more accurately within the context of the model: "Where within the swing should the golfer strike the ball?"

To determine the best position within each swing for striking the ball, the simulation examines a range of points within the swing and tests them all to determine which ball position results in the best flight. For every point where the clubhead is within $4 \mathrm{~cm}$ of its lowest (approximately 900 points per swing), the impact and aerodynamic analysis is performed and the value of the objective function (17) calculated. The point with the highest value is selected as the ideal point of contact for that swing, and that ball carry and objective function value are considered to be the value for that particular swing. For all impact calculations, the ball is assumed to strike the clubface at the projected CoM location.

\subsection{Implementation}

The golfer and club model was implemented in MapleSim 7 [19]. This program generates the equations for multibody dynamic systems. After implementation, the generated equations were then exported to create a Cfunction that calculates the velocity and orientation of the clubhead throughout the swing given the muscle activations and initial conditions for the golfer's joints. The integration is performed using a RungeKutta solver with a timestep of $10^{-5}$ seconds. This function was compiled into a .mex function in Matlab [20] where the optimization process was performed. A single swing simulation took around 10 seconds on an 8 core Intel Xeon CPU at $2.13 \mathrm{GHz}$.

A number of different optimization techniques were attempted using Matlab including fminsearch, patternsearch, and genetic algorithms (ga). In testing, patternsearch 
was best able to avoid local minimums and find the global optimum solution. Also, patternsearch allows for simple parallelization as many simulations can be performed simultaneously within this scheme. The final optimizations were performed on a high performance computer with $16 \mathrm{~GB}$ of RAM and 16 available cores at $2.13 \mathrm{GHz}$. A complete optimization run took between 60 and 120 minute depending on the strength of the initial guess and the desired solutions.

Within the optimization, the activation times of the various torque generators were optimized simultaneously with the starting joint angles for the golfer, for a total of 11 variables within the optimization. These variables along with their constraints and initial guess are shown in Table 8 . In addition, the activation of each joint torque was constrained to occur before the deactivation. The optimization process was stopped when changes to the activations resulted in less than $1 \mathrm{~cm}$ changes to the final carry distance. To increase our confidence that a global optimum had been reached, the optimization for the golfer and club configuration presented in this paper was run for a number of different initial guesses which all reached the same final configuration.

\section{Model Limitations}

There are many elements of the golf swing that have been intentionally left out of the golfer model to decrease its complexity. The most obvious omission is the entire lower body of the golfer. The omitted degrees of freedom include the lateral shifting of the pelvis and the independent rotation of the pelvis with respect to the torso. In the context of this model, the removal of horizontal shifting seems reasonable because the modern golf swing is primarily a rotational movement. While the golfer feels a significant shift of weight from one leg to the other during the swing, the actual translation of the pelvis is quite small during the swing [13].

The independent rotation of the pelvis has been cited as an indicator of golfer excellence [9] but it is not a degree of freedom that is required to capture the kinematics of the hands of the golfer gripping the club. The rotation of the upper torso will have to start earlier if the pelvis rotation is omitted, but the motion of the shoulder joint will remain the same. If the goal of the model was to determine what portion of the power is generated by different joints, this degree of freedom would be important, but since the goal is to evaluate club performance, pelvis rotation can be lumped in with upper torso rotation. This same simplification will also lead to a higher torque for the torso of the golfer as this torque must provide all of the required angular acceleration of the arms.

Another omission from the mechanical structure of the model is the omission of the trailing (right) arm of the golfer. The inclusion of this arm would have resulted in a closed kinematic loop within the model and complicated the equations that must be solved to determine its motion by introducing algebraic constraints to the differential equations. So instead, it is assumed that the golfer's trailing arm plays a negligible role in providing power to the swing and is simply used for stabilization. Since the model golfer does not need to stabilize the swing (the joints used are inherently stable), the second arm is unnecessary. Since the trailing arm has been removed from the golfer, any power production from it must be lumped into the leading arm and its strength has been slightly increased. In particular, the pronation-supination strength is required to be larger to close the clubface.

The joint torque model described in Section 2.1.1 describes how the golfer model is activated in a way that approximates the muscles of a real golfer. This approximation of the muscle activity of the golfer simplifies the model and reduces the number of parameters that must be optimized to control the swing. A higher fidelity alternative is a model that includes individual muscles attached to a skeletal model of the golfer [23]. However, the inclusion of multiple muscles for each of the joints would require the solution of the muscle redundancy problem [4] and greatly increase the length of time required for simulations.

\section{Results and Discussion}

After running the optimization procedure outlined in Section 2.5, the results for a single representative swing were obtained. The following plots show the large range of swing characteristics that can be examined using this model.

First, the optimized ball carry is $195.7 \mathrm{~m}$ (214 yards) with a lateral deviation of $1.8 \mathrm{~m}$. After impact the ball has 3280 rotations per minute (RPM) of backspin, a launch angle of $18.1^{\circ}$, and a ball speed of $60.8 \mathrm{~m} \mathrm{~s}^{-1}$ (136 mph). The trajectory results can be found in Figure 11 corresponding to a slight draw. The higher than expected backspin causes a higher arcing trajectory, but this is the best carry result that can be obtained while satisfying the biomechanical constraints on the golfer's swing.

The clubhead speed is shown in Figure 12. Peak clubhead speed is reached slightly before impact and the clubhead speed at impact is $41.5 \mathrm{~m} \mathrm{~s}^{-1}$ (92.8 $\left.\mathrm{mph}\right)$. 
Table 8 List of the 11 variables that are optimized by the patternsearch and their initial values and constraints. simLength means the end of the simulation time.

\begin{tabular}{llll} 
Variable & Initial Guess & Min. Value & Max. Value \\
\hline Torso Activation & $0 \mathrm{~s}$ & $-0.1 \mathrm{~s}$ & $0.1 \mathrm{~s}$ \\
Torso Deactivation & $0.22 \mathrm{~s}$ & $0.1 \mathrm{~s}$ & simLength $\mathrm{s}$ \\
Shoulder Activation & $0.004 \mathrm{~s}$ & $-0.05 \mathrm{~s}$ & $0.1 \mathrm{~s}$ \\
Shoulder Deactivation & $0.18 \mathrm{~s}$ & $0.05 \mathrm{~s}$ & simLength $\mathrm{s}$ \\
Forearm Activation & $0.13 \mathrm{~s}$ & $0.05 \mathrm{~s}$ & simLength $\mathrm{s}$ \\
Forearm Deactivation & $0.22 \mathrm{~s}$ & $0.05 \mathrm{~s}$ & simLength $\mathrm{s}$ \\
Wrist Activation & $0.04 \mathrm{~s}$ & $0 \mathrm{~s}$ & $0.15 \mathrm{~s}$ \\
Wrist Deactivation & $0.12 \mathrm{~s}$ & $0.1 \mathrm{~s}$ & simLength $\mathrm{s}$ \\
Initial Shoulder JA & $70^{\circ}$ & $68^{\circ}$ & $72^{\circ}$ \\
Initial Forearm JA & $90^{\circ}$ & $88^{\circ}$ & $92^{\circ}$ \\
Initial Wrist JA & $110^{\circ}$ & $108^{\circ}$ & $112^{\circ}$ \\
\hline
\end{tabular}
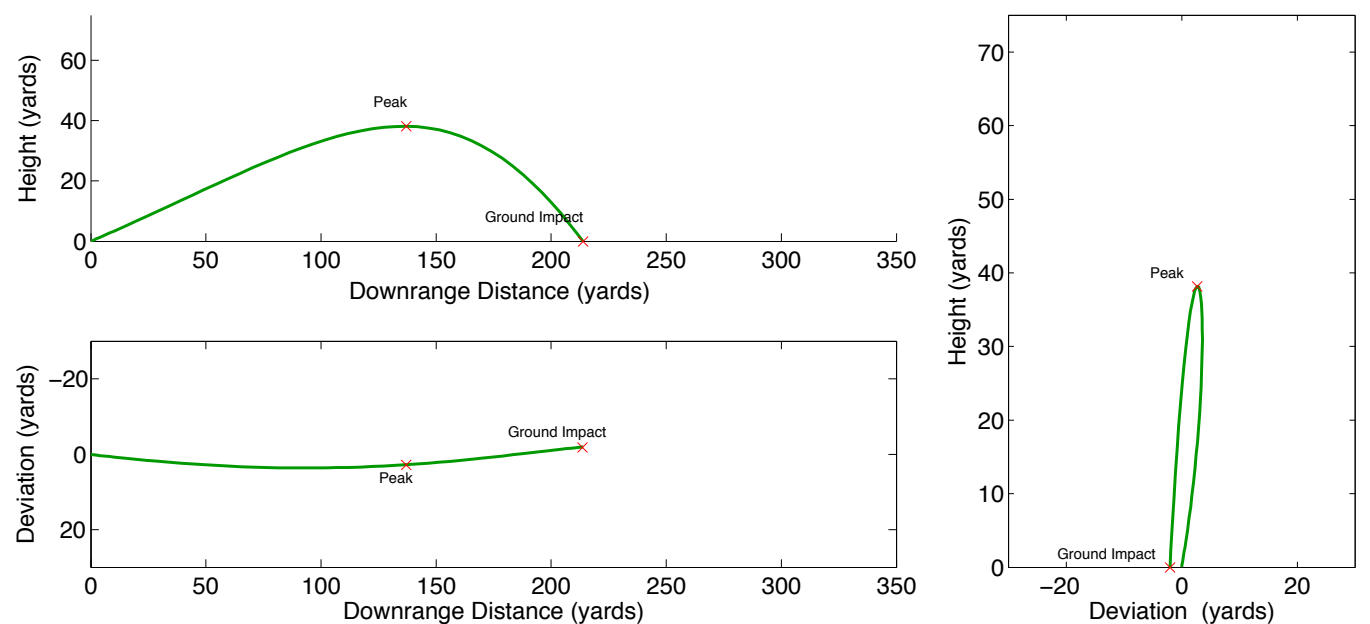

Fig. 11 Ball trajectory for swing with default parameters.

This clubhead speed is similar to that observed by Mackenzie, $41.9 \mathrm{~m} \mathrm{~s}^{-1}$ [16], for a similar model and observed experimentally by Milne \& Davis [24]. The clubhead speed at impact is slightly slower than the peak clubhead speed to achieve better impact conditions with the ball. By delaying the impact slightly, the club has started to move upwards improving the attack angle of the swing and increasing the launch angle.

Figure 14 shows this delay illustrating how the club is moving upwards at impact $\left(v_{y}>0\right)$. Increasing the delay further decreases the benefit since the clubhead is slowing down and clubhead speed is the most significant factor in the ball carry distance. The ball is struck near the low point of the swing at a position about $1 \mathrm{~m}$ along the z-axis in front of the golfer's torso. The ball is struck $1.7 \mathrm{~cm}$ above the low point of the swing at a legal tee height. The inside-out pattern of the golf drive can also be observed in Figure 14 by noting that the velocity in the Z-direction is still positive (moving away from the golfer's body) at impact. The inside-out pattern is suggested by many golf professionals as the best way to hit long straight drives [36]. This pattern also

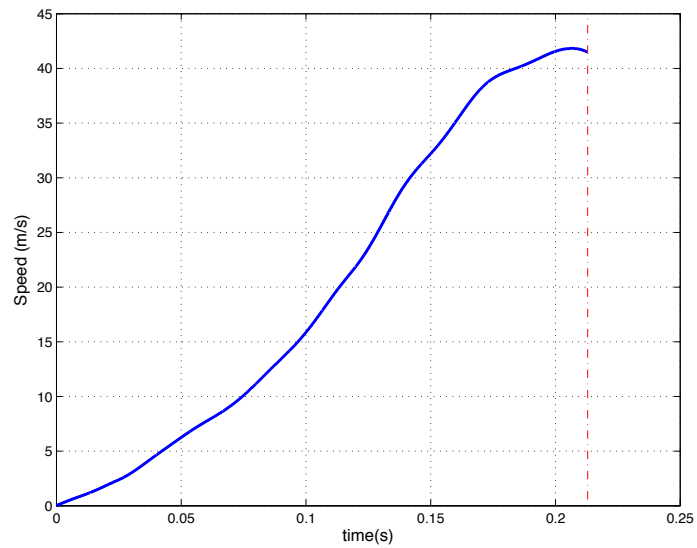

Fig. 12 Clubhead centre of mass speed for swing with default parameters.

matches the observations of Mackenzie [16] using a similar model. At impact, Table 9 compares the clubhead speeds at impact of our model to those found in [16]. The velocity in the $\mathrm{x}$-direction is very similar, but our 
Table 9 Comparison of clubhead velocity at impact $\left(\mathrm{m} \mathrm{s}^{-1}\right)$ of our model to [16]

\begin{tabular}{ccc} 
& Model & Mackenzie \\
\hline$V_{x}$ & 41.2 & 41.9 \\
$V_{y}$ & 3.4 & 6.1 \\
$V_{z}$ & 2.8 & 7.2
\end{tabular}

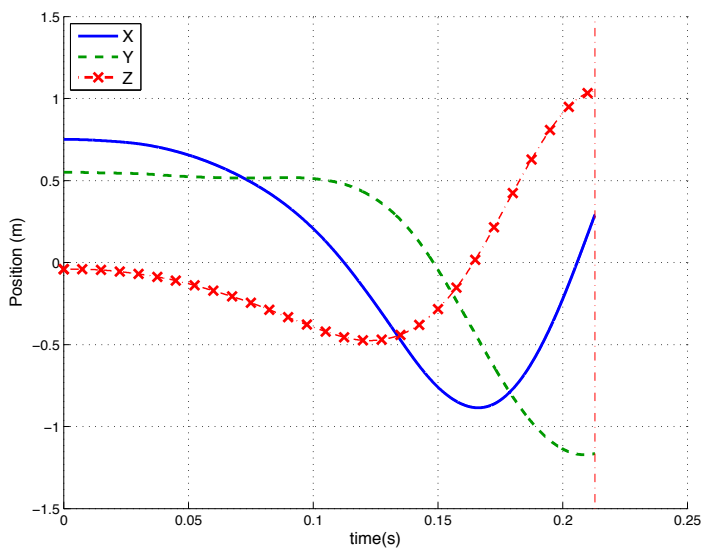

Fig. 13 Clubhead centre of mass position

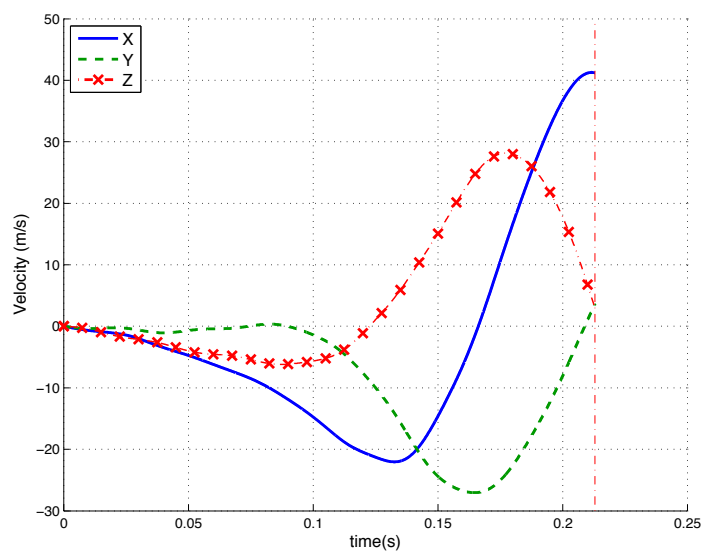

Fig. 14 Clubhead centre of mass velocity

velocities in the $y$ and z-directions are quite a bit lower. This is likely because the inclusion of an impact model penalizes high lateral velocity at impact as too high a velocity will result in off-line trajectories.

The golfer's kinematics are also available from the model. The joint angles are shown in Figure 15. This Figure clearly illustrates the kinematic sequencing of the swing. At $t=0$, the torso starts its forward motion, followed by the shoulder around $t=0.05$, the wrist at $t=0.1$ and finally the forearm around $t=0.15$. This progression from the proximal to distal joints is similar to those found in experimental results [25] and previous modeling results $[16]$.

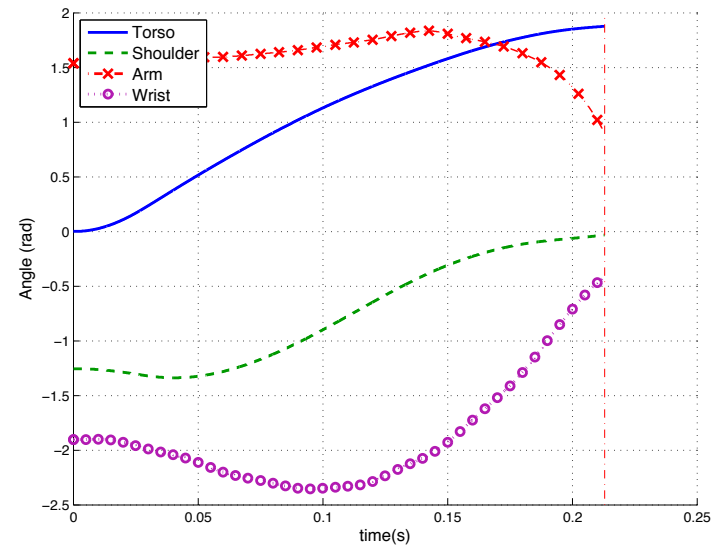

Fig. 15 Golfer joint angles.

The motion of the forearm degree of freedom is interesting and worth discussing further. While the motion of the forearm begins at $t=0.15 \mathrm{~s}$ the there is significant acceleration in the supination of the arm despite declining torque $t=0.17 \mathrm{~s}$. This acceleration occurs because as the wrist joint brings the club into line with the forearm, the effective moment of inertia of the arm about its long axis is reduced as the mass moves closer to the central axis. The speed of rotation then increases until impact. The combination of the passive and active torques for each joint is shown in Figure 16. This Figure shows the joint torque at each joint peaking as the motion begins and falling off as the joint is accelerated.

Figure 17 shows the active portion of the joint torques applied to the model. The torso torque was initiated at $t=0$ and peaked at $164 \mathrm{Nm}$ which is below the maximum reported in the literature [33]. The shoulder torque was initiated at $t=0.022$ and peaked at $91 \mathrm{Nm}$ which is slightly above the reported value in the literature [11]. This is because the maximum shoulder torque was increased to account for including only the lead arm in the swing model. The wrist torque was initiated at $t=0.11$ and deactivated at $t=0.12$. This short activation is enough to help bring the club in line with the arm for the rest of the swing. Finally the forearm torque is activated to square the clubface at $t=0.14$. This torque remains active until $t=0.17$. The sequence of these timings match the experimental timings found in [25].

The model is especially sensitive to changes in the timing of the forearm torque as it is difficult to square the face of the club at impact without precise timing of the forearm torque. Too early a torque will close the face at impact and too late a torque will open the face at 


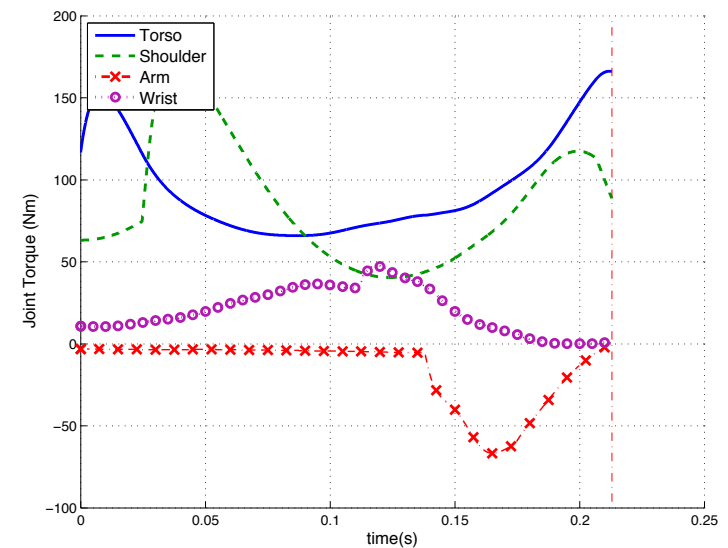

Fig. 16 Total joint torques

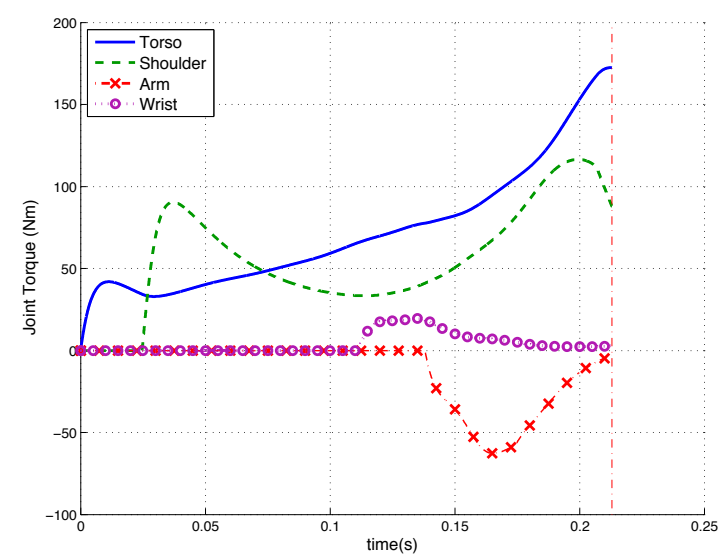

Fig. 17 Active portion of joint torques

impact and both of these scenarios lead to large lateral deviations in the ball trajectory.

Finally, the model is able to incorporate the flexing and bending of the shaft during the swing. This is easiest to see in Figure 18. The forward and backward flexing of the shaft clearly shows the club bend backwards during the swing and then flex forward for impact. This is the expected result as shown in previous experiments [24]. For the representative golfer and shaft, the club is bent forward $4 \mathrm{~cm}$ at impact. The droop oscillates more than expected (possible due to a lack of damping in the shaft model) but does exhibit downward bending (negative droop) at impact. This compares favourably with the experimental results obtained by Sandhu et al. [32] which showed a similar trend in the club droop. Table 10 compares the measured club deflection at impact of the model to Mackenzie [16] and experimental results from Sandhu et al.
Table 10 Comparison of club deflection at impact $(\mathrm{cm})$ to [16] and [32]

\begin{tabular}{rccc} 
Deflection & Model & Mackenzie & Sandhu et al. \\
\hline Lead/Lag & 4.1 & 4.0 & 2.7 to 6.5 \\
Toe-up/Toe Down & -0.8 & -2.0 & -1.3 to 2.7
\end{tabular}

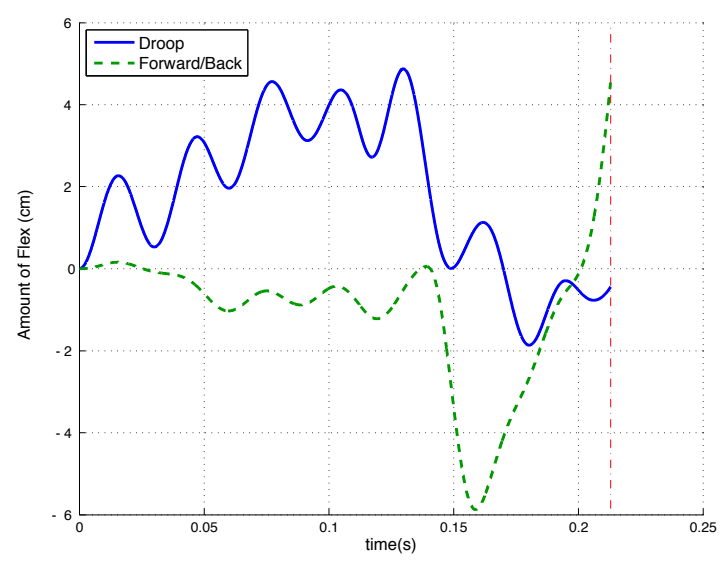

Fig. 18 Club flexing as measured from the grip.

\section{Conclusions}

A three-dimensional, forward dynamic model of the golfer and club that can be evaluated using ball trajectories computed by an impact and aerodynamics model was created and used to simulate a representative golfer and club. The swing of a simulated golfer is optimized to produce the longest ball carry. In addition to combining four separate models into a single comprehensive model, passive joint forces and club aerodynamics were added to the golfer and club model. The model golfer swings with a clubhead speed of $41.5 \mathrm{~m} \mathrm{~s}^{-1}(92.8 \mathrm{mph})$ while striking the ball $196 \mathrm{~m}$ (214 yards). The model golfer's joints follow the expected kinematic chain from the torso rotation starting at $t=0 \mathrm{~s}$, the shoulder at $t=0.03 \mathrm{~s}$, and the wrist at $t=0.12 \mathrm{~s}$.

The combined model is a significant contribution to our ability to test golf club design parameters and golf swing biomechanics in simulatio as it could be used to answer many questions of interest in golf club design. By changing the parameters of the club head, the effects of clubhead mass, moment of inertia, and centre of mass position of the clubhead can be investigated. Similarly, other club parameters including the length and stiffness properties of the shaft could be investigated.

Acknowledgements We thank Mike Stachura of Golf Digest for providing robot testing results carried out by Gene Parente of Golf Laboratories. Financial support by the Natural Sciences and Engineering Research Council of Canada is also gratefully acknowledged. 


\section{References}

1. Audu, M., Davy, D.: The influence of muscle model complexity in musculoskeletal motion modeling. Journal of biomechanical engineering 107(2), 147-57 (1985)

2. Betzler, N.F.: The Effect of Differing Shaft Dynamics on the Biomechanics of the Golf Swing. Ph.D. thesis, Edinburgh Napier University (2010)

3. Cochran, A., Stobbs, J.: Search for the Perfect Swing, 2nd edn. Triumph Books, Chicago (2005)

4. Crowninshield, R.D., Brand, R.A.: A physiologically based criterion of muscle force prediction in locomotion. Journal of Biomechanics 14(11), 793-801 (1981)

5. Engin, A., Chen, S.M.: Statistical Data Base for the Biomechanical Properties of the Human Shoulder Complex - II:Passive Resistive Properties Beyond the Shoulder Complex Sinus. Journal of biomechanical engineering 108(3), 222-227 (1986)

6. Henrikson, E., Wood, P., Hart, J.: Experimental investigation of golf driver club head drag reduction through the use of aerodynamic features on the driver crown. Procedia Engineering 72, 726-731 (2014)

7. Hirashima, M., Ohgane, K., Kudo, K., Hase, K., Ohtsuki, T.: Counteractive relationship between the interaction torque and muscle torque at the wrist is predestined in ball-throwing. Journal of Neurophysiology 90(3), 1449-63 (2003)

8. Iwatsubo, T., Adachi, K., Kitagawa, T.: A study of link models for dynamic analysis of swing motion. In: S. Ujihashi, S.J. Haake (eds.) Engineering of Sport 4, pp. 701707. Blackwell Publishing, Kyoto (2002)

9. Joyce, C., Burnett, A., Ball, K.: Methodological considerations for the $3 \mathrm{D}$ measurement of the X-factor and lower trunk movement in golf. Sports Biomechanics - International Society of Biomechanics in Sports 9(3), 206-21 (2010)

10. Kenny, I.C., McCloy, A.J., Wallace, E.S., Otto, S.R.: Segmental sequencing of kinetic energy in a computersimulated golf swing. Sports Engineering 11(1), 37-45 (2008)

11. Kuhlman, J., Ianotti, J., Kelly, M., Riegler, F., Gevaert, M., Ergin, T.: Isokinetic and isometric measurement of strength of external rotation and abduction of the shoulder. Journal of Bone and Joint Surgery 74(9), 1320-1333 (1992)

12. Lampsa, M.A.: Maximizing Distance of the Golf Drive: An Optimal Control Study. Journal of Dynamic Systems, Measurement, and Control 97(4), 362 (1975)

13. Lindsay, D.M., Mantrop, S., Vandervoort, A.A.: A Review of Biomechanical Differences Between Golfers of Varied Skill Levels. International Journal of Sports Science and Coaching 3, 187-197 (2009)

14. MacKenzie, S.J.: Understanding the Role of Shaft Stiffness in the Golf Swing. Ph.D. thesis, University of Saskatchewan (2005)

15. MacKenzie, S.J., Sprigings, E.J.: A three-dimensional forward dynamics model of the golf swing. Sports Engineering (Springer Science \& Business Media B.V.) 11(4), $165-175(2009)$

16. MacKenzie, S.J., Sprigings, E.J.: Understanding the role of shaft stiffness in the golf swing. Sports Engineering 12(1), 13-19 (2009)

17. MacKenzie, S.J., Sprigings, E.J.: Understanding the mechanisms of shaft deflection in the golf swing. Sports Engineering 12(2), 69-75 (2010)

18. Mansour, J.M., Audu, M.L.: The Passive Elastic Moment at the Knee and its Influence on Human Gait. Journal of Biomechanics 19(5), 369-373 (1986)
19. MapleSim: Version 6.4. MapleSoft, Waterloo, ON (2014)

20. MATLAB: Version 8.2.0.701 (R2013b). The MathWorks Inc., Natick, Massachusetts (2013)

21. McGill, S., Seguin, J., Bennett, G.: Passive stiffness of the lumbar torso in flexion, extension, lateral bending, and axial rotation. Effect of belt wearing and breath holding. Spine 19(6), 696-704 (1994)

22. McPhee, J.J., Andrews, G.C.: Effect of sidespin and wind on projectile trajectory, with particular application to golf. American Journal of Physics 56(10), 933 (1988)

23. Mehrabi, N., Razavian, R.S., McPhee, J.: A PhysicsBased Musculoskeletal Driver Model to Study Steering Tasks. Journal of Computational and Non-Linear Dynamics (2014)

24. Milne, R.D., Davis, J.P.: The role of the shaft in the golf swing. Journal of biomechanics 25(9), 975-983 (1992)

25. Neal, R., Lumsden, R., Holland, M., Mason, B.: Body Segment Sequencing and Timing in Golf. International Journal of Sports Science \& Coaching 2(0), 25-36 (2007)

26. Nesbit, S.M.: A three dimensional kinematic and kinetic study of the golf swing. Journal of Sports Science \& Medicine 4, 499-519 (2005)

27. Nigg, B.M., Herzog, W.: Biomechanics of the Musculoskeletal System, 3rd edn. John Wiley \& Sons, Chichester (2006)

28. Petersen, W., McPhee, J.: Comparison of ImpulseMomentum and Finite Element Models for Impact between Golf Ball and Clubhead. In: Science and Golf V: Proceedings of the World Scientific Congress of Golf. Phoenix, USA (2008)

29. Pickering, W.M., Vickers, G.T.: On the double pendulum model of the golf swing. Sports Engineering 2, 161-172 (1999)

30. Quintavalla, S.J.: A generally applicable model for the aerodynamic behavior of golf balls. In: E. Thain (ed.) Science and Golf IV: Proceedings of the 2002 World Scientific Congress of Golf, pp. 341-348. Routledge, St. Andrews, Scotland (2002)

31. Reyes, M., Mittendorf, A.: A Mathematical Swing Model for a Long-Driving Champion. In: M.R. Farrally, A.J. Cochran (eds.) Science and golf III: proceedings of the 1998 World Scientific Congress of Golf, pp. 13-19. Human Kinetics, St. Andrews, Scotland (1999)

32. Sandhu, S., Millard, M., McPhee, J., Brekke, D.: 3D dynamic modelling and simulation of a golf drive. Procedia Engineering 2(2), 3243-3248 (2010)

33. Schultz, a., Cromwell, R., Warwick, D., Andersson, G.: Lumbar trunk muscle use in standing isometric heavy exertions. Journal of orthopaedic research : official publication of the Orthopaedic Research Society 5(3), 320-329 (1987). DOI 10.1002/jor.1100050303

34. Sharp, R.S.: On the mechanics of the golf swing. Proceedings of the Royal Society A: Mathematical, Physical and Engineering Sciences 465(2102), 551-570 (2009)

35. Shi, P., McPhee, J.: Dynamics of flexible multibody systems using virtual work and linear graph theory. Multibody System Dynamics 4, 355-381 (1999)

36. Suttie, J.: How to fix a faulty swing path. Golf Magazine 51(8), 55 (2009)

37. Vena, A., Budney, D., Forest, T., Carey, J.: Sports Engineering (Springer Science \& Business Media B.V.) 13(3) 105-123 (2011)

38. Yamaguchi, G.: Dynamic Modeling of Musculoskeletal Motion. Springer Science and Business Media, New York NY (2006) 